\title{
Amplitude envelope synchronization in coupled chaotic oscillators
}

\author{
J. M. Gonzalez-Miranda \\ Departamento de Fisica Fundamental, Universidad de Barcelona, Avenida Diagonal 647, 08028 Barcelona, Spain
}

(Received 17 August 2001; revised manuscript received 30 November 2001; published 7 March 2002)

\begin{abstract}
A peculiar type of synchronization has been found when two Van der Pol-Duffing oscillators, evolving in different chaotic attractors, are coupled. As the coupling increases, the frequencies of the two oscillators remain different, while a synchronized modulation of the amplitudes of a signal of each system develops, and a null Lyapunov exponent of the uncoupled systems becomes negative and gradually larger in absolute value. This phenomenon is characterized by an appropriate correlation function between the returns of the signals, and interpreted in terms of the mutual excitation of new frequencies in the oscillators power spectra. This form of synchronization also occurs in other systems, but it shows up mixed with or screened by other forms of synchronization, as illustrated in this paper by means of the examples of the dynamic behavior observed for three other different models of chaotic oscillators.
\end{abstract}

DOI: 10.1103/PhysRevE.65.036232

PACS number(s): 05.45.Xt, 05.45.Pq

\section{INTRODUCTION}

The dynamics of two coupled, chaotic oscillators is an issue of great interest in science and engineering where one often deals with two or more nonlinear units (e.g., lasers, electric circuits, and neurons) that are mutually coupled. Likewise, it is interesting for application to the understanding of cooperative behaviors of networks of chaotic systems. Fundamental results are the reports on two mutually coupled chaotic oscillators given by Fujisaka and Yamada [1] on identical synchronization (IS) of identical systems, and by Rosenblum, Pikovsky, and Kurths [2] on phase synchronization (PS) of nonidentical systems. PS and IS have also been studied in unidirectionally coupled chaotic systems [3,4], and PS has been studied in periodically driven chaotic systems [5]. PS has been an object of great attention in the last years, in particular, regarding its application to the synchronization of biological rhythms (see, for example, Refs. [6,7] and references therein). In most cases this form of synchronization means the locking of the frequencies of some oscillating variables of the coupled oscillators while the signal amplitudes remain uncorrelated.

The present paper reports on a numerical study of the dynamics of two different diffusively coupled Van der PolDuffing oscillators [8]. This is interesting because this system exhibits a form of synchronization in which, as the coupling strength increases, a new frequency is forced into the spectrum of the two oscillators, which is responsible for long time variations in the amplitude envelopes of the time evolutions of the system variables. These long term amplitude variations are correlated because the peaks corresponding to the new frequencies occur at the same value, and develop simultaneously in the two oscillators with the coupling strength. This synchronization behavior will be called amplitude envelope synchronization (AES). The Van der PolDuffing oscillators studied here happen to be special in the sense that AES is the only synchronization behavior that is observed. In other systems, such as the Rossler model [9], AES has been found so weak that it is screened by PS; while, there are systems such as the circuit of Chua and co-workers [10], and the Proto-Lorenz system [11], in which both AES and PS have been observed combined. The development of AES starts at very small coupling strengths; moreover, it is signaled by a null Lyapunov exponent of the uncoupled systems becoming negative, as it occurs with PS. Because of this, besides its interest as a special form of synchronization, the development of AES, combined with PS, appears as a good candidate to explain certain disagreements between the coupling strengths at which the Lyapunov exponent becomes null and at which the onset of PS occurs; which can be found in the literature (see $[5,12]$ and references therein).

This paper is then arranged as follows. In Sec. II, the phenomenology of amplitude envelope synchronization is presented, in Sec. III the dynamic mechanism that causes AES is discussed, in Sec. IV the manifestation of AES combined with PS is demonstrated, and finally, Sec. V is devoted to discussion and summary.

\section{AMPLITUDE ENVELOPE SYNCHRONIZATION}

The system studied is a set of two diffusively coupled Van der Pol-Duffing oscillators, labeled 1 and 2, whose equations of motion, written in dimensionless form, are [8]

$$
\begin{gathered}
\dot{x}_{1,2}=-100\left(x_{1,2}^{3}-0.35 x_{1,2}-y_{1,2}\right), \\
\dot{y}_{1,2}=x_{1,2}-y_{1,2}-z_{1,2}+\frac{\varepsilon}{2}\left(y_{2,1}-y_{1,2}\right), \\
\dot{z}_{1,2}=\beta_{1,2} y_{1,2},
\end{gathered}
$$

with $\beta_{1}$ and $\beta_{2}$ representing parameters of the system and $\varepsilon$ a measure of the coupling strength. To study the synchronization of the two coupled chaotic oscillators as a function of $\varepsilon$, the parameter values have been fixed in $\beta_{1}=610$ and $\beta_{2}$ $=650$, which for $\varepsilon=0$ correspond to two oscillators evolving in different chaotic attractors such that their dynamics is a coherent rotation around a unique center [13]. Moreover, the structural stability of the results obtained has been successfully tested by additional studies performed for $\beta_{1}$ $=600$ and $\beta_{2}=620$, and for $\beta_{1}=600$ and $\beta_{2}=680$. 
Previous reports on the dynamic behavior of two Rossler models mutually [2] and unidirectionally coupled [4] and a Rossler model periodically driven [5], reveal the development of PS as the intensity of the coupling increases. In these investigations, PS is characterized by means of an average frequency for each chaotic oscillator that can be defined as $\Omega_{1,2}=2 \pi /\left\langle T\left(s_{1,2}\right)\right\rangle,\left\langle T\left(s_{1,2}\right)\right\rangle$ being the average value of the return times for a given signal of the systems, $s_{1,2}(t)$ [i.e., the times elapsed between successive maxima of $\left.s_{1,2}(t)\right]$, or in other equivalent forms appropriate to characterize the evolution of the phase $\phi$ of the rotation around the center of the attractor by means of the condition $\Omega=\langle d \phi / d t\rangle$ [5]. PS is seen as the fulfilling of the condition $m \Omega_{2}-n \Omega_{1}=0$, with $m$ and $n$ integers, when the coupling reaches a certain critical value. Moreover, PS is signaled in the Lyapunov spectrum by a null exponent becoming negative, corresponding to the stability of the locking of these frequencies.

Two coupled Van der Pol-Duffing oscillators behave in a quite different way as illustrated in Fig. 1. The increase in $\varepsilon$ leaves almost unaltered the values of the frequencies [Fig. 1(a)] that undergo changes of about $2 \%$ while its difference stays roughly between 0.6 and 0.7 . The Lyapunov spectrum, whose four largest exponents are shown in Fig. 1(b), is of the type $(+,+, 0,0,-,-)$ for $\varepsilon=0$, and becomes of the type $(+,+, 0,-,-,-)$ apparently in the same way as in the PS described in previous studies [2,4-6]. However, a closer look at the inset suggests that the fourth Lyapunov exponent, which is one of two null exponents of the uncoupled systems, is negative, although very small in absolute value for any $\varepsilon \gtrsim 0$. So in this case we do not have the PS, described by the condition $\Delta \Omega=\left|\Omega_{2}-\Omega_{1}\right|=0$ and observed in Refs. [2,4,5,7], nor a resonance with $m$ and $n$ small integers as those reported in Ref. [5] with $m, n \leqslant 2$ and in Ref. [6] where $m, n \leqslant 5$; however, the Lyapunov spectrum suggests the development of some type of locking between the two systems, which develops from $\varepsilon \gtrsim 0$.

To characterize this synchronization, the dynamics of the two coupled Van der Pol-Duffing oscillators has been analyzed in appropriate Poincaré sections. From time evolutions of $z_{1,2}(t)$, large enough to have a reliable description of the dynamics, the times of occurrence of relative maxima $\tau_{1}^{(i)}$ and $\tau_{2}^{(j)}$, and the values of the corresponding maxima $Z_{1}^{(i)}$ and $Z_{2}^{(j)}$, are recorded for $i=1,2, \ldots, N_{1}$ and $j$ $=1,2, \ldots, N_{2}$. The results are two discrete functions $Z_{1}(\tau)$ and $Z_{2}(\tau)$, with values defined at $\tau=\tau_{1}^{(i)}$ and $\tau=\tau_{2}^{(j)}$. For the coupled Van der Pol-Duffing oscillators studied here, a correlation between $Z_{1}(\tau)$ and $Z_{2}(\tau)$ develops as $\varepsilon$ increases, which has been called here AES. This is a form of synchronization between the maxima achieved by $z_{1}(t)$ and $z_{2}(t)$, but not between the times of occurrence of these maxima nor between the signals themselves. AES can be measured by means of correlation coefficients $r_{2,1}$ and $r_{1,2}$ properly defined to take into account that $Z_{1}(\tau)$ and $Z_{2}(\tau)$ are given at different times, and that the two signals we look for correlations to have different shapes. Given $Z_{1}(\tau)$ and $Z_{2}(\tau)$, the surrogate of $Z_{1}(\tau)$ relative to $Z_{2}(\tau)$ is defined as the ordered set of points

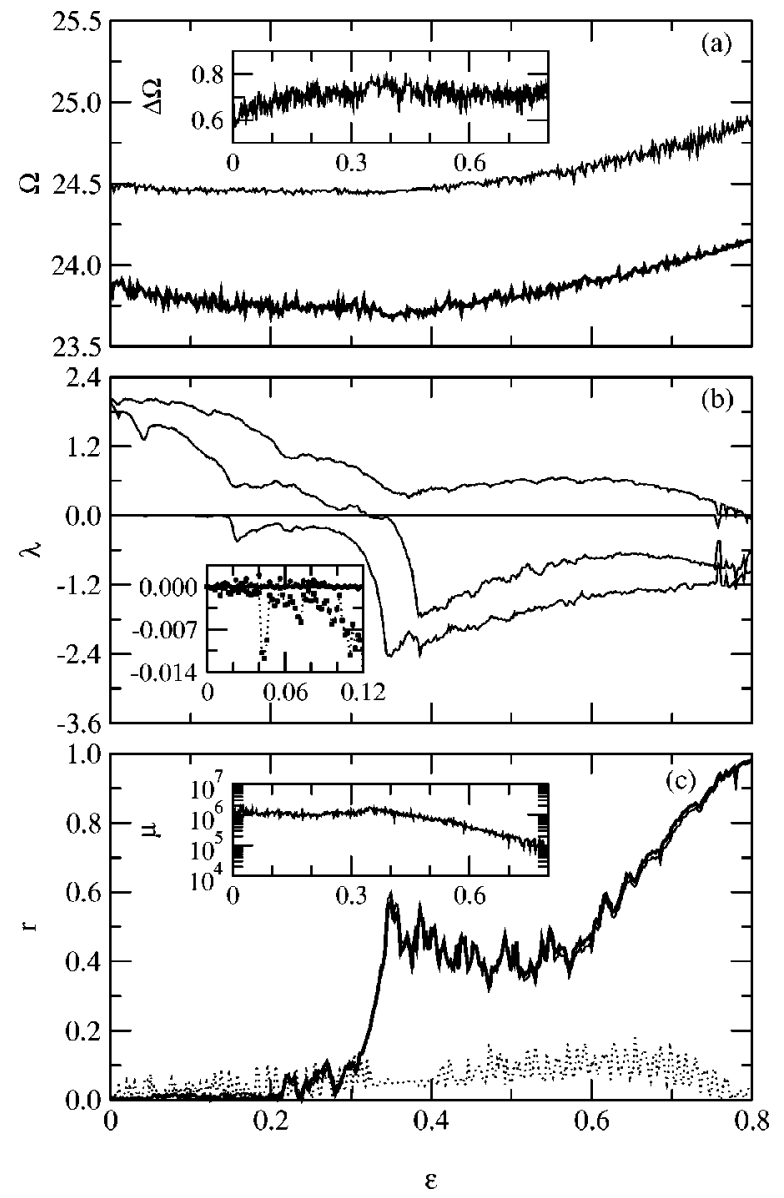

FIG. 1. Dynamic behavior of two coupled Van der Pol-Duffing oscillators as the coupling strength increases. (a) Frequencies $\Omega_{1}$ (thick line) and $\Omega_{2}$ (thin line) and their difference $\Delta \Omega$ $=\left|\Omega_{2}-\Omega_{1}\right|$, displayed in the inset; (b) the four largest Lyapunov exponents (the inset displays a detail of the third and fourth Lyapunov exponents for small values of $\varepsilon$ ); and (c) plots of the Spearman correlation coefficients $r_{2,1}$ (thick line), $r_{1,2}$ (thin line), and $r$ (dotted line). The inset shows the dependence of $\varepsilon$ for the mutually false nearest neighbor parameter computed from a sample of size $N=20000$, obtained after running the system for 30000 time steps to avoid transitories. All quantities plotted are dimensionless.

$$
S_{1}^{(k)}=\frac{Z_{1}^{(i)}\left(\tau_{1}^{(i+1)}-\tau_{2}^{(k)}\right)+Z_{1}^{(i+1)}\left(\tau_{2}^{(k)}-\tau_{1}^{(i)}\right)}{\left(\tau_{1}^{(i+1)}-\tau_{1}^{(i)}\right)}
$$

with $i$ given by the condition $\tau_{1}(i) \leqslant \tau_{2}(k)<\tau_{1}(i+1)$ and $k \in \mathcal{A}_{2,1}$ with

$$
\mathcal{A}_{2,1}=\left\{j \mid j=1,2, \ldots, N_{2} \text { and } \tau_{1}^{(1)} \leqslant \tau_{2}^{(j)}<\tau_{1}^{\left(N_{1}\right)}\right\}
$$

Then, the correlation of $Z_{2}(\tau)$ to $Z_{1}(\tau)$ is quantified by the Spearman rank-order correlation coefficient [14] between $S_{1}(k)$ and $Z_{2}(k)$ and is given by 


$$
r_{2,1}=\frac{\sum_{k \in \mathcal{A}_{2,1}}\left(\sigma_{1}^{(k)}-\bar{\sigma}_{1}\right)\left(\zeta_{2}^{(k)}-\bar{\zeta}_{2}\right)}{\sqrt{\sum_{k \in \mathcal{A}_{2,1}}\left(\sigma_{1}^{(k)}-\bar{\sigma}_{1}\right)^{2}} \sqrt{\sum_{k \in \mathcal{A}_{2,1}}\left(\zeta_{2}^{(k)}-\bar{\zeta}_{2}\right)^{2}}},
$$

$\sigma_{1}^{(k)}$ and $\zeta_{2}^{(k)}$ being the ranks of $S_{1}^{(k)}$ and $Z_{2}^{(k)}$ in the corresponding sets of values defined by $S_{1}^{(j)}$ and $Z_{2}^{(j)}$ with $j$ $\in \mathcal{A}_{2,1}$, and $\bar{\sigma}_{1}$ and $\bar{\zeta}_{2}$ their average values. The correlation coefficient $r_{1,2}$, can be defined in the same way, and will provide the same information as $r_{2,1}$.

These correlation coefficients are displayed in Fig. 1(c) together with the Spearman rank-order correlation coefficient $r$ for $z_{1}(t)$ and $z_{2}(t)$, which does not require the use of surrogate data. This last correlation shows that $z_{1}(t)$ and $z_{2}(t)$ can be considered only as very weakly correlated signals. The behavior of $r_{2,1}$ and $r_{1,2}$ is quite different: it displays an increasing correlation between $Z_{1}(\tau)$ and $Z_{2}(\tau)$ with coefficients $r_{2,1} \approx r_{1,2}>0.2$ for $\varepsilon>0.32$, and $r_{2,1} \approx r_{1,2}$ $\lesssim 1$ for $\varepsilon \lesssim 0.8$. The main features of the dependence of $r_{2,1}$ and $r_{1,2}$ on $\varepsilon$ are in correspondence with the dynamic events displayed by the Lyapunov spectrum. The qualitative behavior of $r_{2,1}$ and $r_{1,2}$ appears to be mainly the result of a competition between $\lambda_{4}$ whose increase in absolute value reinforces the stability of the synchronization, and the positive exponents that indicate a high degree of chaos and then a destruction of correlations. One observes an increase in the correlation when there is an increase of $\left|\lambda_{4}\right|$ combined with a decrease of the positive exponents. To be more specific, the following features are stressed. For $\varepsilon \leqslant 0.15$, the system is hyperchaotic, having two decreasing positive Lyapunov exponents, while $\lambda_{4}$ shows very small increase in absolute value $\left(-0.02<\lambda_{4}<0\right)$, so they show no noticeable correlation. For $0.15<\varepsilon \leqslant 0.22$, the fourth exponent starts to be noticeable, but one of the positive exponents $\left(\lambda_{2}\right)$ stops decreasing, and the correlation is still unnoticeable. For 0.22 $<\varepsilon \leqslant 0.36, \lambda_{1}$ and $\lambda_{2}$ decrease, with $\lambda_{2}$ changing sign and leaving the system chaotic, while $\left|\lambda_{4}\right|$ increases notoriously; then, it is in this region where the correlation is greatly enhanced. For $0.36<\varepsilon \leqslant 0.50, \lambda_{1}$ increases (i.e., the system becomes more chaotic), while $\left|\lambda_{4}\right|$ steadily decreases, and some correlation is lost. Finally, a new increase in the correlation is obtained for $0.50<\varepsilon \leqslant 0.80$, when $\lambda_{1}$ decreases to zero while $\lambda_{4}$ is almost stationary.

The nature of the above correlation in the peak-to-peak dynamics between the two coupled Van der Pol-Duffing oscillators can be visualized by looking directly to plots of $Z_{1,2}(\tau)$ at different values of $\varepsilon$ as illustrated in Fig. 2. There it is seen how the two functions follow a synchronized oscillation of the same frequency which is disrupted by bursts that momentarily destroy the synchronization. This correlation, that does not exist for $\varepsilon=0$, results almost unnoticeable at $\varepsilon=0.12$, and well developed at $\varepsilon=0.74$. For the system variables $z_{1,2}(t)$, this means a synchronized modulation of their amplitudes, but because there is no locking between $\Omega_{1}$ and $\Omega_{2}$ the amplitudes themselves are not correlated and $z_{1}(t)$ and $z_{2}(t)$ evolve almost independently from each other. This is why the name AES was given.

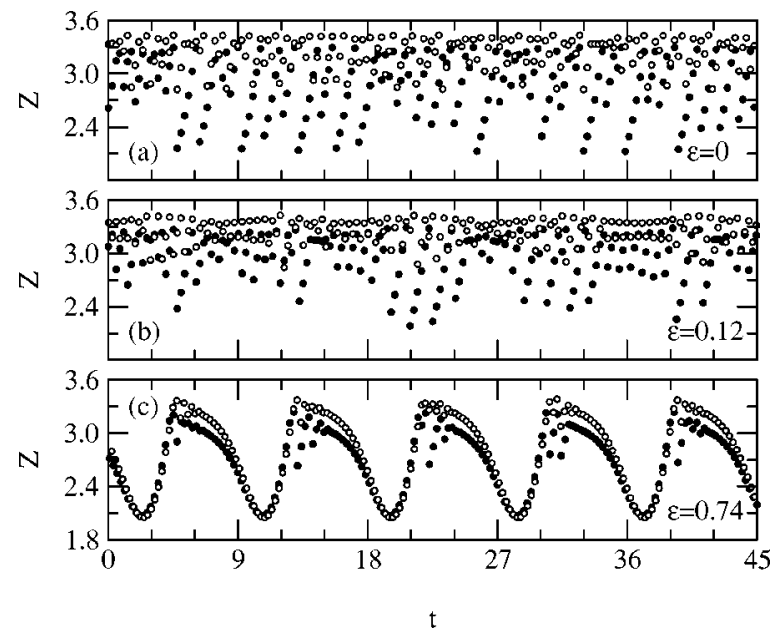

FIG. 2. Time evolution of the returns $Z_{1}(\tau)$ (filled circles) and $Z_{2}(\tau)$ (open circles) at increasing values of $\varepsilon$. All quantities plotted are dimensionless.

Besides PS, there is another form of synchronization between nonidentical systems named generalized synchronization (GS) [15]. This has been mainly studied in the context of directionally coupled chaotic systems; i.e., when a drive system acts on a response system. GS is defined as the existence of a synchronization condition of the type $\mathbf{r}_{R}(t)=\phi\left[\mathbf{r}_{D}(t)\right]$, with $\phi[\cdot]$ representing a functional relation that determines the phase space trajectory of the response $\mathbf{r}_{R}(t)$ from the trajectory of the drive $\mathbf{r}_{D}(t)$. When the transition to GS occurs the maximum conditional Lyapunov exponent changes its sign from positive to negative. Because AES experiences its major growth after a positive exponent has become negative, one might ask if AES is a special form of GS that occurs in mutually coupled chaotic oscillators, verifying the condition $\mathbf{r}_{1}(t)=\phi\left[\mathbf{r}_{2}(t)\right]$, with $\phi[\cdot]$ representing an invertible relation, and $\mathbf{r}_{1,2}(t)$ the trajectories of the systems that are mutually coupled. However, just a zero crossing of one positive Lyapunov exponent is not enough to assess that there is GS; for example, such an event in the Lyapunov spectra has been reported in Ref. [16] as a signal for a transition from PS to lag synchronization (a form of synchronism that may develop on phase synchronized systems [16]). Moreover, there have been reports on the observation of PS between two three-dimensional systems mutually coupled with just one positive exponent [12]. Therefore, to study the possibility of GS, a standard test has been done by computing the mutually false nearest neighbor parameter [15] as a function of $\varepsilon$. Given the vectors of phase space variables of the two oscillators, $\mathbf{r}_{1,2}\left(t_{k}\right)=\left[x_{1,2}\left(t_{k}\right), y_{1,2}\left(t_{k}\right), z_{1,2}\left(t_{k}\right)\right]$ with $k=1,2, \ldots, N$, this parameter is computed by means of

$$
\mu=\frac{1}{N} \sum_{k=1}^{N} \frac{\left|\mathbf{r}_{2}\left(t_{k}\right)-\mathbf{r}_{2}\left(t_{k}^{(1)}\right)\right|}{\left|\mathbf{r}_{1}\left(t_{k}\right)-\mathbf{r}_{1}\left(t_{k}^{(1)}\right)\right|} \frac{\left|\mathbf{r}_{1}\left(t_{k}\right)-\mathbf{r}_{1}\left(t_{k}^{(2)}\right)\right|}{\left|\mathbf{r}_{2}\left(t_{k}\right)-\mathbf{r}_{2}\left(t_{k}^{(2)}\right)\right|},
$$

where $t_{k}^{(1,2)}$ is the time label of the nearest neighbor of $\mathbf{r}_{1,2}\left(t_{k}\right)$, and $|\cdot|$ the Cartesian distance. If there is GS, $\mu$ is a number close to 1 ; if not, it is a large number whose magnitude is comparable to the product of the size of the attractors 

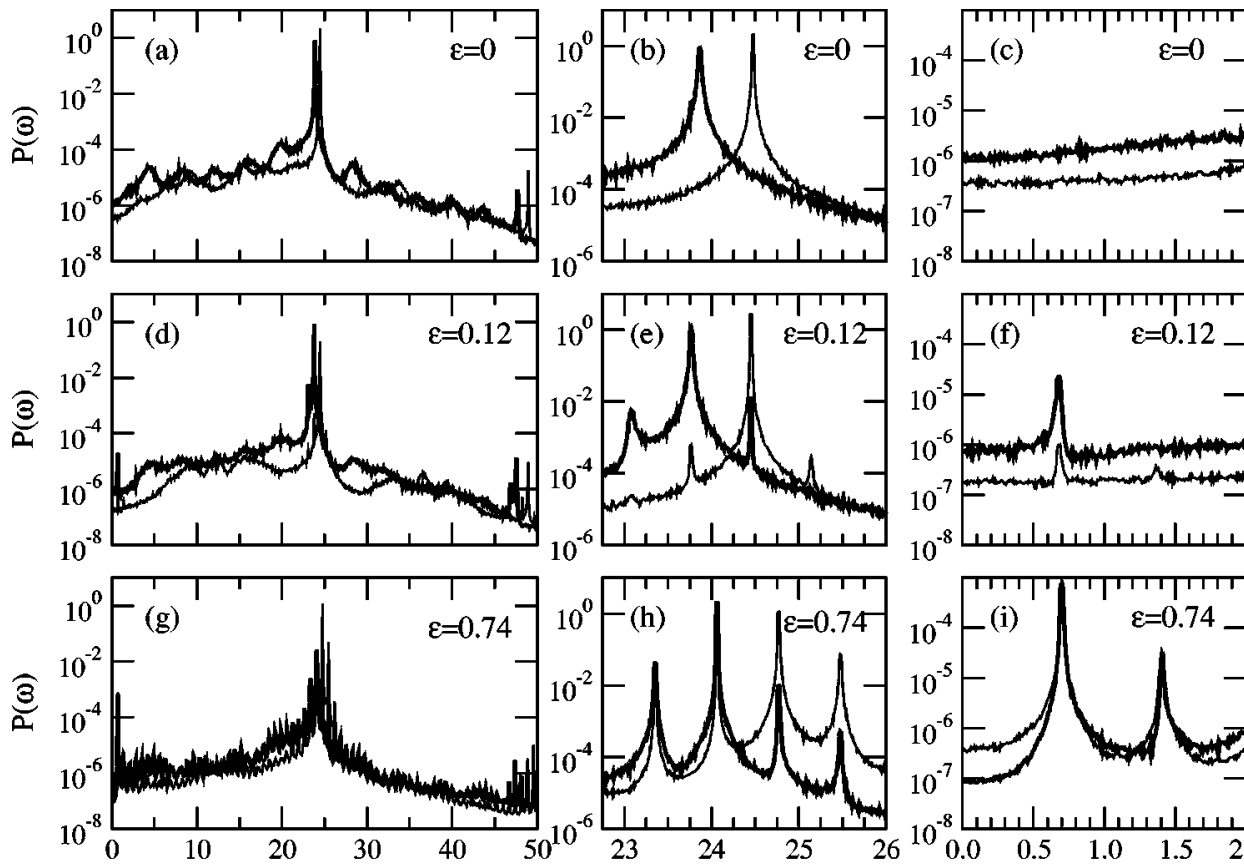

$\boldsymbol{\omega}$

$\mathbf{\omega}$

divided by the product of the distances between nearest neighbors in the time series, $\mathbf{r}_{1,2}\left(t_{k}\right)$. Because this is a statistical test of local neighborliness between two time series, it is still valid for the case of mutual coupling. The results for $\mu$, displayed in the inset of Fig. 1(c) show that although being a decreasing function of $\varepsilon$, it stays always several orders of magnitude far from 1 , and never more than one order of magnitude from the product of the sizes of the attractors divided by the product of the distances between nearest neighbors; therefore, GS has to be discarded. In fact, these results are consistent with those for the Spearman rank-order correlation coefficient between $z_{1}(t)$ and $z_{2}(t)$, shown in Fig. 1(c), which, despite having a mild increase with $\varepsilon$, indicate practically no correlation between the time evolutions of these signals. The proper interpretation of the behavior of the Lyapunov spectrum here is that the fourth Lyapunov exponent is the one measuring AES. The decrease (and zero crossings) of the positive exponents in this case just reveals a destruction of chaos as a result of the variation of $\varepsilon$, which being a parameter of the six-dimensional system may change the chaoticity of its dynamics, as other parameters do.

\section{DYNAMIC MECHANISM FOR AES}

AES can be understood by means of the analysis of the one-sided power spectral density [14] of $z_{1,2}(t)$,

$$
P_{1,2}(\omega)=2\left|\int_{0}^{\infty} z_{1,2}(t) e^{i \omega t} d t\right|^{2},
$$

which has been computed and analyzed at 41 values of $\varepsilon$, between 0 and 0.8 . Some of the results for $P_{1,2}(\omega)$ are displayed in Fig. 3 for values of $\varepsilon$ corresponding to: no synchronization at all (first row), very mild synchronization (second row), and well-developed synchronization (third
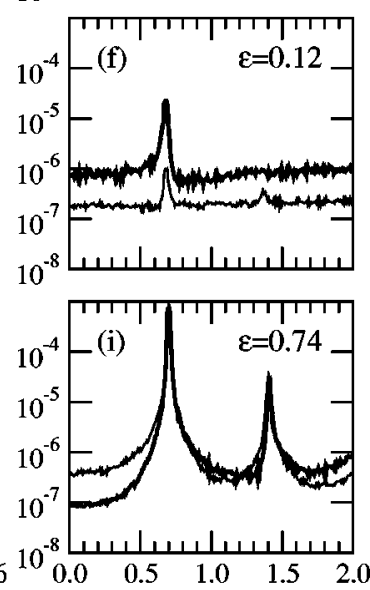

$\omega$ tities plotted are dimensionless.
FIG. 3. Power spectral densities for $z_{1}(t)$ (thick line) and $z_{2}(t)$ (thin line) at several values of the coupling strength, displayed in different regions of $\omega$ : in the first column there are shown all relevant frequencies, in the second the region around the main maxima, and in the third the very small frequencies. All quantities plotted are dimensionless.

row). Moreover, the most relevant features of the power spectra are summarized in Fig. 4. The power spectra of the uncoupled oscillators [Figs. 3(a)-3(c)] have well-defined main frequencies at $\omega_{1}^{(M)}$ and $\omega_{2}^{(M)}$ and secondary sharp peaks at their harmonics $2 \omega_{1}^{(M)}$ and $2 \omega_{2}^{(M)}$. The effect of mild coupling [Figs. 3(d) and 3(e) and 4(a)] in each system is to enhance the movements with frequencies corresponding to

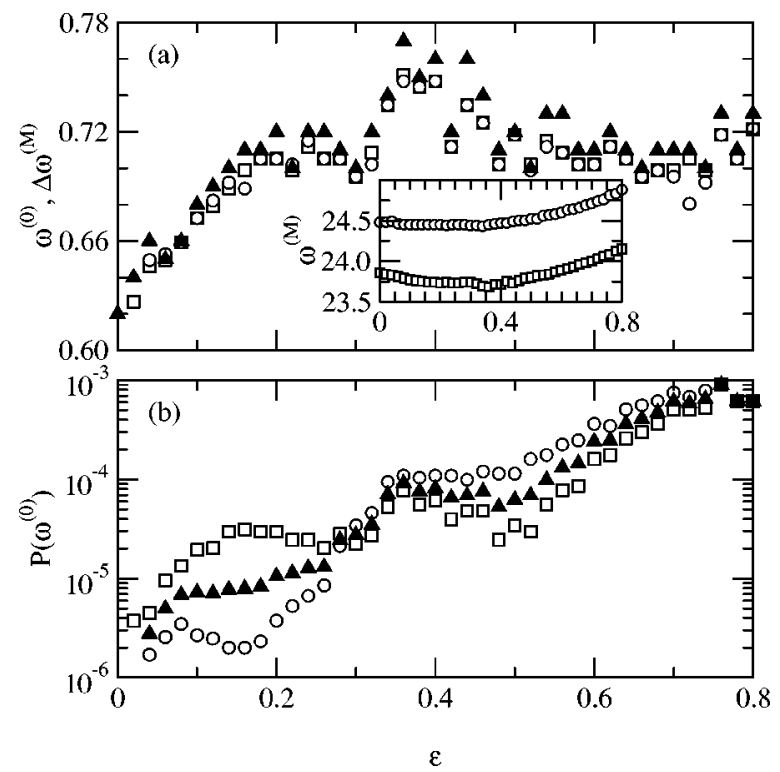

FIG. 4. Main features of the power spectra of $z_{1}(t)$ (squares) and $z_{2}(t)$ (circles) as functions of the coupling strength. (a) The frequencies of the first peaks $\omega_{1,2}^{(0)}$ together with $\Delta \omega^{(M)}=\omega_{2}^{(M)}$ $-\omega_{1}^{(M)}$ (triangles), with $\omega_{1,2}^{(M)}$ the frequencies of the main peaks displayed in the inset, and (b) the height of the first peaks $P_{1,2}\left(\omega^{(0)}\right)$, as well as its geometric mean $m_{P}$ (triangles). All quan- 
the main frequencies of the other system; that is, when the coupling is switched on, the maxima occurring at $\omega_{1}^{(M)}$ and $\omega_{2}^{(M)}$ create new secondary peaks at $\omega_{1}^{\prime}=\omega_{2}^{(M)}$ for subsystem 1 and $\omega_{2}^{\prime}=\omega_{1}^{(M)}$ for subsystem 2. Moreover, the harmonics of the series to which $\omega_{1}^{\prime}$ and $\omega_{2}^{\prime}$ belong are also excited; then, being $\omega_{2}^{(M)}>\omega_{1}^{(M)}$, it happens that a new frequency appears at $\omega_{1}^{(0)} \lesssim \omega_{2}^{(M)}-\omega_{1}^{(M)}=\Delta \omega^{(M)}$ for subsystem 1 [Fig. 3(f)]. As the coupling continues to increase, this frequency induces a new peak in subsystem $2, \omega_{2}^{(0)}=\omega_{1}^{(0)}$. For further increase of the coupling [Figs. 3(g)-3(i) and 4(b)] this new common frequency that becomes the fundamental frequency of each spectrum, is enhanced in the two systems, this being the cause for the development of AES. The behavior of the geometric mean of the heights of the first peaks $m_{P}$ $=\sqrt{P_{1}\left(\omega^{(0)}\right) P_{2}\left(\omega^{(0)}\right)}$, deserves to be noted: this quantity is a combined measure of how developed are the peaks that correspond to $\omega_{1}^{(0)}$ and $\omega_{2}^{(0)}$, so that its dependence on $\varepsilon$ should be in accordance with that of the $r_{2,1}$ and $r_{1,2}$, and with the Lyapunov spectrum described above. The comparison of Figs. 1(c) and 4(b) illustrates that this is indeed the case.

The development of new frequencies in the dynamics of the oscillators is the key to understand AES. The dynamic mechanism for the appearance of new frequencies that applies here has been studied recently by Pikovsky et al. [5]. Basically, it happens that if an external periodic forcing, with a frequency $\Omega$ acts on a chaotic system, it results in a modification of its dynamics whose primary manifestation is the growth of new discrete components in the power spectra at frequencies that include $\Omega$. This mechanism is illustrated in Fig. 5 for a Van der Pol-Duffing oscillator driven by a periodic force of amplitude $A$ and frequency $\Omega$ as follows:

$$
\begin{gathered}
\dot{x}=-100\left(x^{3}-0.35 x-y\right), \\
\dot{y}=x-y-z+A \sin (\Omega t), \\
\dot{z}=610 y .
\end{gathered}
$$

For $A$ fixed at a small value, and several values of $\Omega$ given around the dominant spectral peak, Fig. 5(a) shows as a first effect of the forcing the appearance of a new peak at $\Omega$. Moreover, the system being highly coherent with a dominant frequency $\omega^{(M)}$, it happens that now the distance between consecutive peaks happens to be $\left|\Omega-\omega^{(M)}\right|$ so that a new series of harmonics develop that has its first peak at $\left|\Omega-\omega^{(M)}\right|$. This is the dynamic mechanism behind AES. On the other hand, results for fixed $\Omega$ and $A$ changing over four orders of magnitude are displayed in Fig. 5(b). For very mild interaction the spectra is practically unchanged. A mild interaction results in the scenario that has been described in Fig. 5(a). The dynamic mechanisms for PS are observed after further increases in the interaction. For a moderate intensity of the coupling, there is an increase in the number of harmonics that are noticeable, the main peak widens and the background noise near $\omega=0$ increases. For still stronger coupling, $\Omega$ becomes the dominant spectral peak. These mechanisms have to work for two coupled chaotic oscilla-

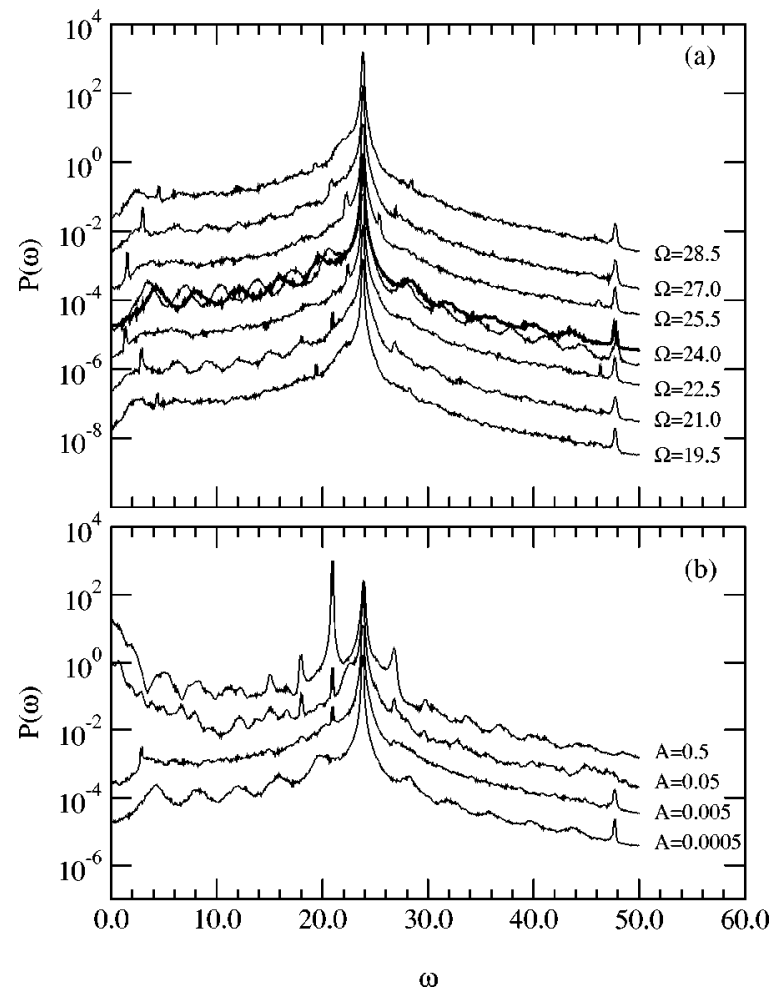

FIG. 5. Effect of a single $\delta$ peak of frequency $\Omega$ and amplitude $A$ forced on the dynamics of the Van der Pol-Duffing oscillator. (a) Action of $\Omega$ for small amplitude $(A=0.01)$. The frequencies (indicated in the figure) take values $\Omega=19.5,21.0, \ldots, 28.5$, and the respective functions $P(\omega)$ have been rescaled by factors $10^{-3}, 10^{-2}, \ldots, 10^{3}$, for the clarity of the figure. The spectrum for the free oscillator appears as a thick line. (b) Effect of $A$, for fixed frequency $(\Omega=21.0)$ with the plots rescaled by factors $10^{0}, \ldots, 10^{3}$ for the amplitudes $A=0.005, \ldots, 0.5$ indicated in the figure.

tors, with the sharper peaks of each spectra playing the role of $\delta$ peaks applied to the other system. However, in this case we have several peaks that are not perfect $\delta$ functions, and there is feedback, so we are faced with a more complex scenario.

The dynamic behavior presented here for a Van der PolDuffing oscillator, with a parameter mismatch of about $6 \%$ appears very different than that reported in Ref. [2] for Rossler oscillators, under a parameter mismatch of 3\%, despite the two systems having a proper rotation structure. However, the two behaviors are not so different when studied in Fourier space as demonstrated in Fig. 6 where the process of phase synchronization is shown for precisely the same case studied in Ref. [2], i.e.,

$$
\begin{gathered}
\dot{x}_{1,2}=-\omega_{1,2} y_{1,2}-z_{1,2}+C\left(x_{2,1}-x_{1,2}\right), \\
\dot{y}_{1,2}=\omega_{1,2} x_{1,2}+0.15 y_{1,2}, \\
\dot{z}_{1,2}=0.2+z_{1,2}\left(x_{1,2}-10\right),
\end{gathered}
$$

with $\omega_{1,2}=1 \pm 0.015$ and $C$ the coupling strength. In Fig. 6 there appears numerical results for $P(\omega)$ and the Lyapunov 


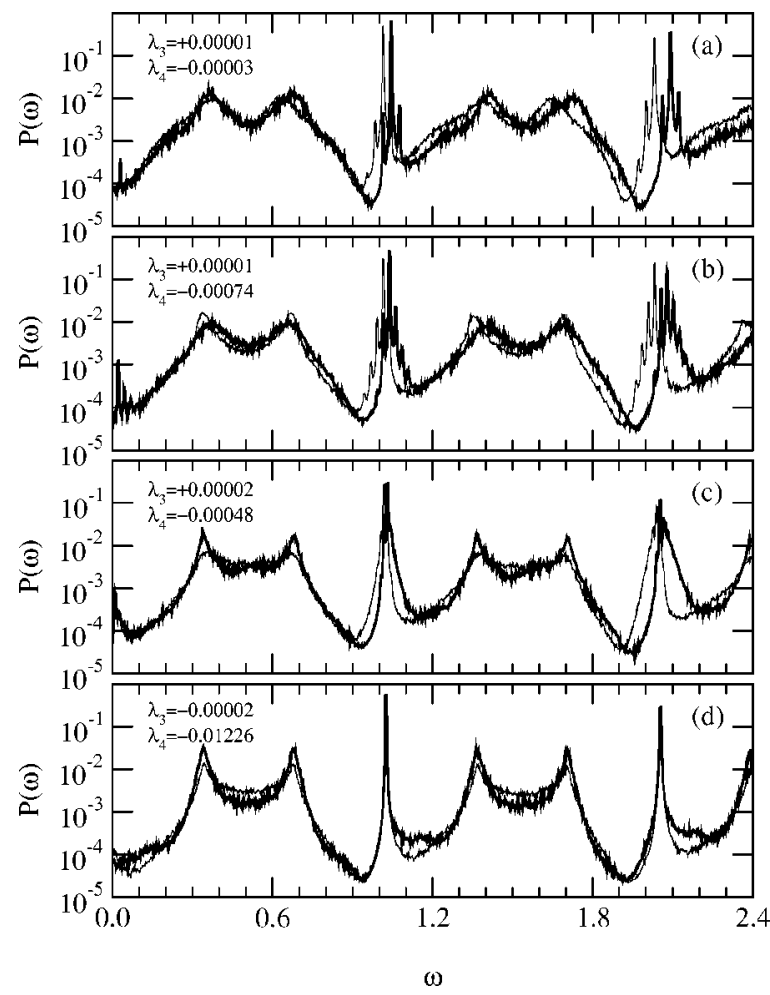

FIG. 6. Fourier space study of the phase synchronization of two coupled Rossler systems with $\omega_{1}=0.985$ (thin line) and $\omega_{2}$ $=1.015$ (thick line) for increasing coupling strength: (a) $C=0.010$, (b) $C=0.020$, (c) $C=0.026$, and (d) $C=0.030$. The corresponding numerical results obtained for the third (null) and fourth Lyapunov exponents have been included in this figure.

exponents computed here for the transition to PS, that occurs at $C \approx 0.028$, as shown in Fig. 3 of Ref. [2]. In this case, the chaotic spectral densities present two main differences with the Van der Pol-Duffing oscillator: for one side the structure of harmonics is stronger here, with secondary peaks of almost the same height as the main peak of each series; moreover, the background noise between peaks is also more structured. However, for mild coupling [Figs. 6(a) and 6(b)] one observes essentially the same type of structures as in Figs. 3 and 5(a): excitation of new peaks around the dominant peaks of the spectra and its harmonics as well as in the neighborhood of $\omega=0$. Together with the observation of a fourth Lyapunov exponent which, although very small in absolute value, is the first negative exponent of the spectrum and these are the signals of AES. In this case, these are hardly noticeable because there are two positive Lyapunov exponents [2] (we are here in the same scenario described above for two coupled Van der Pol-Duffing oscillators when $\varepsilon$ $<0.22$ ). The development of PS, as $C$ increases [Figs. 6(b) and $6(\mathrm{c})$ ] occurs by a combination of the broadening of the peaks with the increase in the number of induced peaks, which results in the shift of the main peak of each spectrum (and its harmonics) to match the corresponding peak of the other [Fig. 6(d)]. This scenario bears great resemblance with that shown in Fig. 5(b) for driving intensities moderate and up, this being the main difference that in this case there is a feedback between the two systems, which lets them lock to each other. Therefore, the Fourier space observation of the dynamics of the two types of oscillators reveals the same underlying mechanism operating in different forms because of the different properties of each system. In particular, the fact that chaos is destroyed faster in the coupled Van der Pol-Duffing oscillators than that in the coupled Rossler systems is essential for the manifestation of AES in the first case, and for its screening in the second. The fast destruction of chaos in the coupled Van der Pol-Duffing oscillators (at $\varepsilon=0.8$ there are no positive Lyapunov exponents) is what prevented the development of PS (and possibly, for even stronger coupling, lag synchronization) in this case.

From the above results it follows that, because different nonlinear systems, even being coherent, have different spectral structures (as well as other properties), and because changes in the parameters of different systems may have different effects on the resulting attractors, the particular dynamic behaviors to be observed in systems of two coupled nonlinear oscillators may have some diversity. This will be illustrated further in the following section by means of the study of two different systems showing all PS and AES in different degrees.

\section{AES AND PS COMBINED}

A system for which AES has been observed combined with PS is the circuit of Chua and co-workers [10]. The equations modeling the system of coupled oscillators, written in dimensionless form, are

$$
\dot{x}_{1,2}=\alpha_{1,2}\left[y_{1,2}-F\left(x_{1,2}\right)\right]
$$

$$
\begin{gathered}
\dot{y}_{1,2}=x_{1,2}-y_{1,2}+z_{1,2}+\frac{\varepsilon}{2}\left(y_{2,1}-y_{1,2}\right), \\
\dot{z}_{1,2}=-\beta_{1,2} y_{1,2},
\end{gathered}
$$

where $F\left(x_{1,2}\right)=a_{1,2} x_{1,2}+b_{1,2}\left(\left|x_{1,2}+1\right|-\left|x_{1,2}-1\right|\right)$. For the numerical study presented here the parameter values used have been $\alpha_{1}=8.65, \beta_{1}=14.29, a_{1}=0.28$, and $b_{1}=$ -0.210 for circuit 1 , and $\alpha_{2}=8.10, \beta_{2}=14.87, a_{2}=0.32$, and $b_{2}=-0.295$ for circuit 2 . These parameters correspond to particular circuits studied experimentally in [10] and [17], respectively; except for $\alpha_{1,2}$, which has been changed to have two disjoint chaotic attractors where the main motion for each oscillator is a rotation around a single center, instead of a double scroll. The variables $z_{1,2}(t)$ have also been used as observables in this case and only the attractors for which $z_{1,2}(t)$ is mostly negative have been considered.

The results for $\Omega_{1,2}(\varepsilon)$ and for the Lyapunov spectrum $\lambda_{i}(\varepsilon)$, displayed in Figs. 7(a) and 7(b), show that two different regimes develop successively as the coupling strength increases, before chaos is destroyed. For very weak coupling strength $(0<\varepsilon \lesssim 0.190)$ we have Regime I in which the phase frequencies $\Omega_{1,2}$ remain close to those of the uncoupled circuits (up to $2 \%$ ) while the fourth Lyapunov exponent, which was null at $\varepsilon=0$, becomes negative and in- 

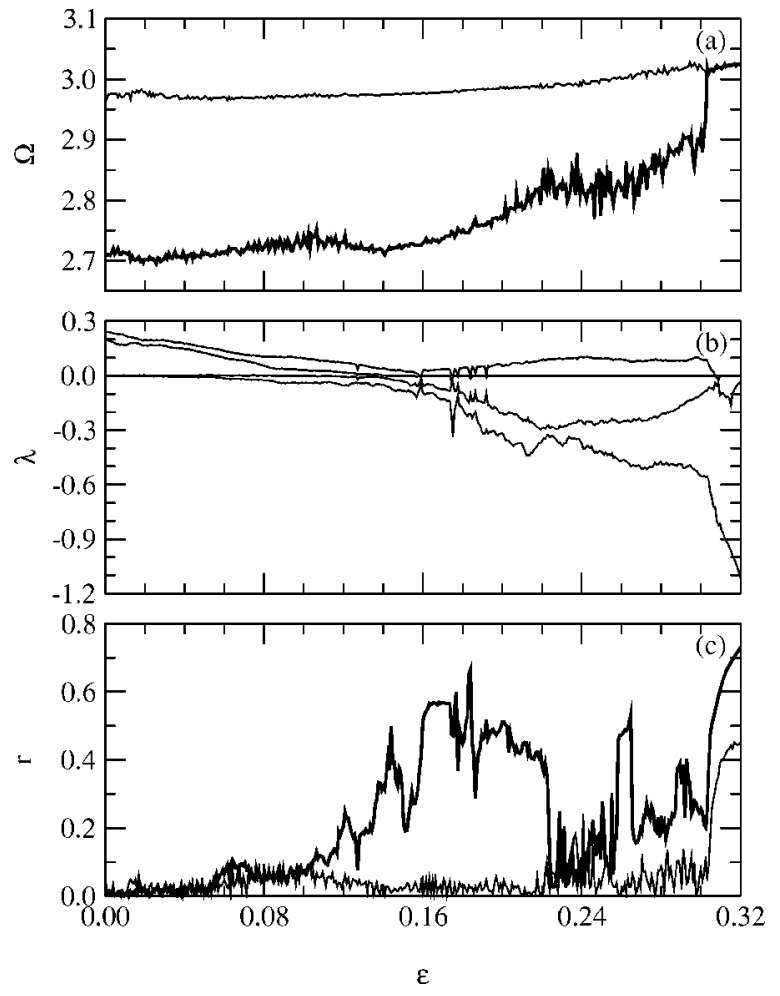

FIG. 7. Dynamic behavior of two coupled Chua circuits as displayed by (a) the phase frequencies $\Omega_{1}$ (thick line) and $\Omega_{2}$ (thin line), (b) the four largest Lyapunov exponents, and (c) the Spearman correlation coefficients between the envelopes $r_{2,1}$ (thick line) and between the signals $r$ (thin line). All quantities plotted are dimensionless.

creases steadily in absolute value for practically any value of $\varepsilon>0$. The system stays chaotic having at least one positive Lyapunov exponent. For weak coupling $(0.190 \leq \varepsilon \leq 0.303)$ Regime II develops with a fast drift of $\Omega_{1}$, which changes its value by more than $10 \%$ leading to a regular phenomenon of phase synchronization at a frequency $\Omega_{1}=\Omega_{2} \approx 3.02$. A faster increase of $\left|\lambda_{4}\right|$ is observed in Regime II, the system being chaotic with one positive Lyapunov exponent. Nearly after PS has been reached all exponents become nonpositive at $\varepsilon \gtrsim 0.308$, being two of them null; i.e., the resulting nonchaotic motion is quasiperiodic.

It is to be noted that the indication, given by $\lambda_{4}<0$ in Regime I, of the development of some locking between the two systems before the onset of PS. The results of the calculation of the Spearman rank-order correlation coefficient $r_{2,1}(\varepsilon)$, displayed in Fig. 7(c), show how this correlation develops within Regime I reaching its maximum values around $\varepsilon \approx 0.18$. The correlation decays at the beginning of Regime II, to experience a new enhancement when the coupling strength increases from $\varepsilon \approx 0.22$, when AES and PS develop combined. The correlation coefficient between the signals themselves, $r(\varepsilon)$, is small until PS is reached; then, it increases notoriously indicating an important degree of correlation between the two systems.

The behavior described above has also been analyzed in terms of the evolution of the one-sided power spectral den-

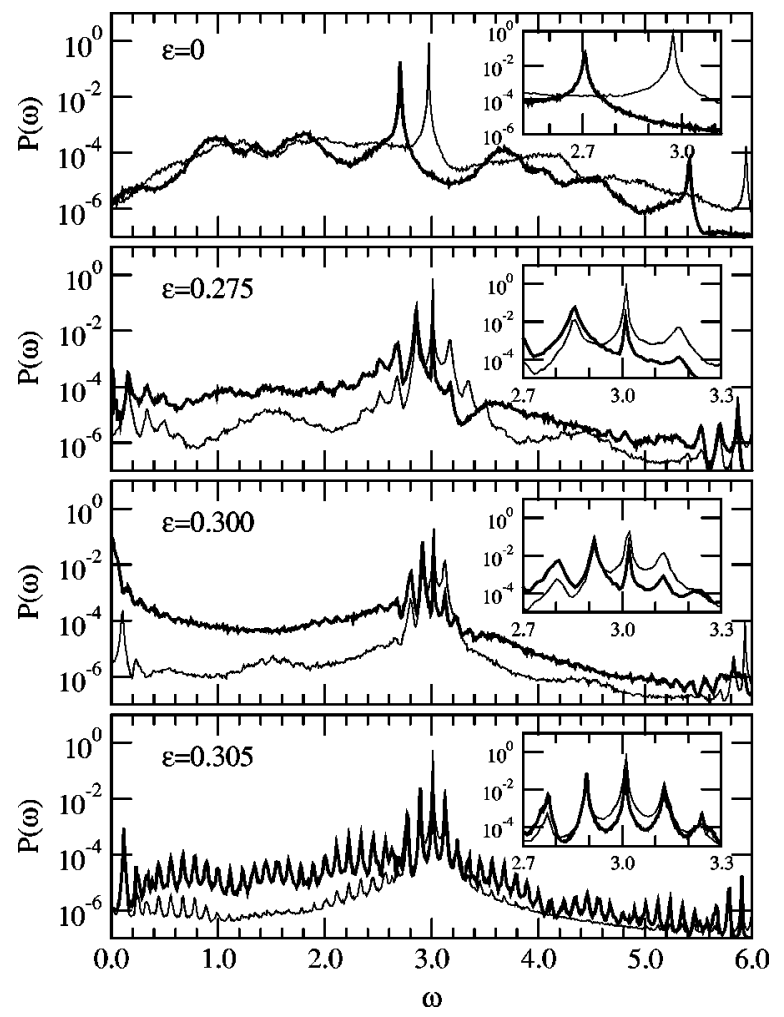

FIG. 8. Power spectral densities for two weakly coupled Chua circuits within Regime II. The thick line is for subsystem 1 and the thin line for subsystem 2 . The insets show in more detail the regions of frequencies around those of the motion of the phase. The first plot, for the uncoupled systems, is given for reference. All quantities plotted are dimensionless.

sity of $z_{1,2}(t)$. The development of new peaks at frequencies around $\omega_{1,2}^{(0)} \approx 0.01$ (while the main peak frequencies governing the dynamics of the phase are close to $\omega_{1,2}^{(M)} \approx 3.0$ ) has been observed. This is shown in Fig. 8 that illustrates the combined development of AES and PS in Regime II as the

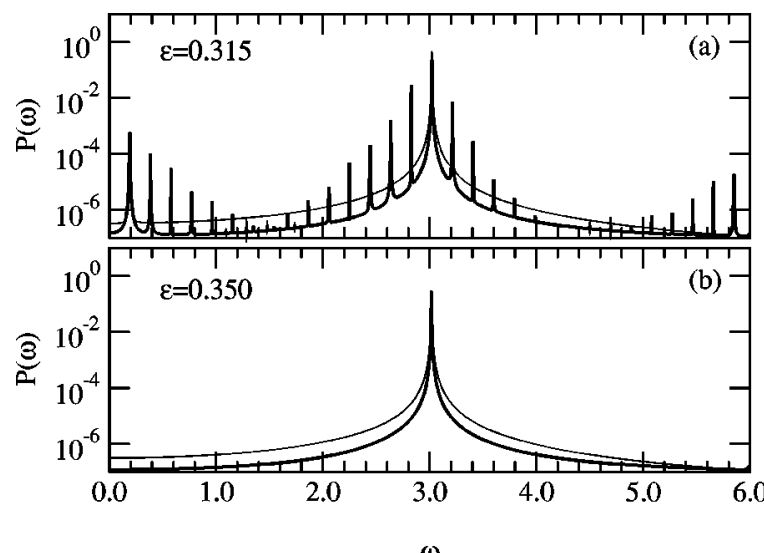

FIG. 9. Power spectra of two coupled Chua circuits at values of the coupling strength where the motion is not chaotic: (a) quasiperiodic motion and (b) periodic motion. The thick line is for subsystem 1 and the thin line for subsystem 2. All quantities plotted are dimensionless. 

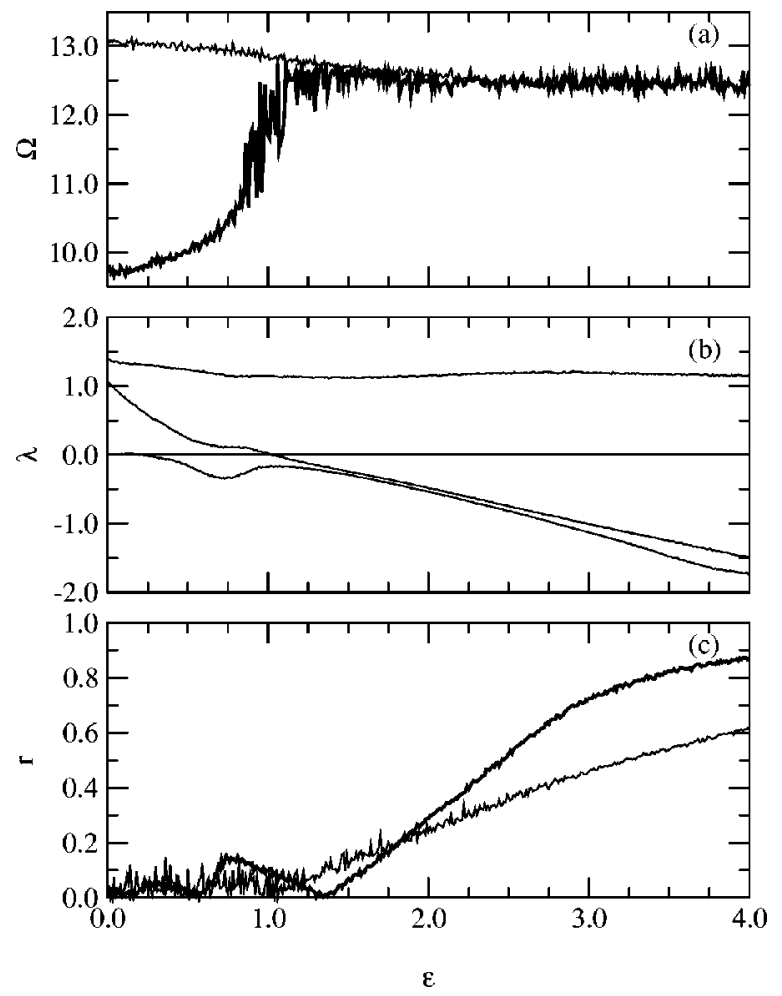

FIG. 10. Dynamic behavior of two coupled Proto-Lorenz systems as displayed by (a) the phase frequencies $\Omega_{1}$ (thick line) and $\Omega_{2}$ (thin line), (b) the four largest Lyapunov exponents, and (c) the Spearman correlation coefficients between the envelopes $r_{2,1}$ (thick line) and between the signals $r$ (thin line). All quantities plotted are dimensionless.

enhancement of the new peaks at $\omega^{(0)}$ that give rise to AES, while the structure of the spectra around $\omega^{(M)}$ rearrange to display the same pattern, giving rise to PS. The corresponding plot for Regime I is not displayed because it is not qualitatively distinct from that in Fig. 3 for the Van der PolDuffing oscillator. After Regime II, chaos has been suppressed, but the new peaks associated to AES combined with the main peaks that dominate PS do persist giving a synchronized quasiperiodic motion as illustrated in Fig. 9(a). Further increase in $\varepsilon$ was found to make the systems periodic (around $\varepsilon \approx 0.326$ ) as the peaks at $\omega_{1}^{(0)}=\omega_{2}^{(0)}$ vanish [a sample of the corresponding spectra appear in Fig. 9(b)].

This set of results shows that Regime I is dominated by AES and it is essentially the same as that described above for the Van der Pol-Duffing oscillator. This synchronized behavior nearly fades out at the transition to Regime II. Within that regime it turns to develop again, simultaneously with PS, so that the amplitude envelopes and phases of the two signals become correlated at the same time leading to a state of enhanced synchronism. This combination of AES and PS does not persist any longer as $\varepsilon$ increases in the periodic regime.

Other chaotic oscillators for which we can observe AES and PS combined is the Proto-Lorenz system. This is a mathematical model introduced by Miranda and Stone [11]. Its chaotic attractor has the structure of a single loop and by
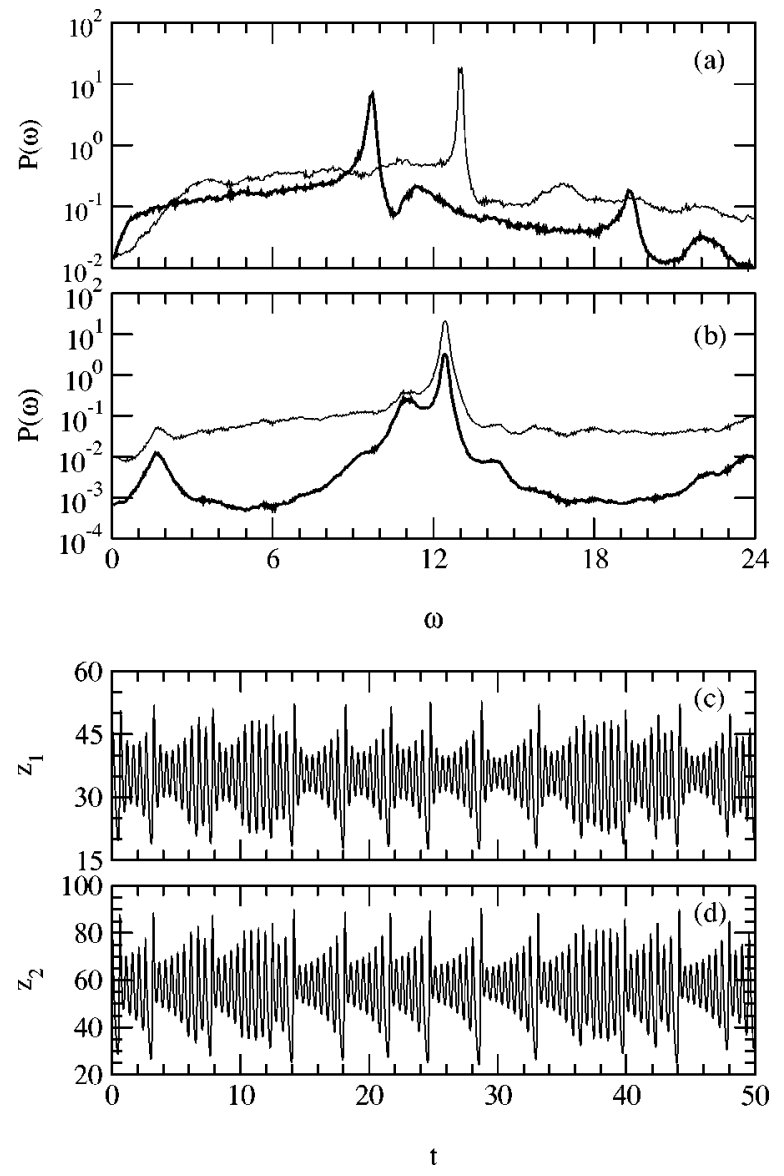

FIG. 11. Power spectra of two coupled Proto-Lorenz systems for (a) $\varepsilon=0$ and (b) $\varepsilon=2.4$. The thick line is for subsystem 1 and the thin line for subsystem 2. Samples of time evolution, at the same coupling strength, of the signals (c) $z_{1}(t)$ and (d) $z_{2}(t)$. All quantities plotted are dimensionless.

appropriate coverings, described in Ref. [11], one can construct symmetric $n$-looped attractors from it, the 2-looped attractor being the well known Lorenz model [18]. The equations of motion for two coupled Proto-Lorenz systems studied here are

$$
\begin{gathered}
\dot{x}_{1,2}=-11 x_{1,2}+\left(10-r_{1,2}\right) y_{1,2}+9 N_{1,2}+y_{1,2} z_{1,2}, \\
\dot{y}_{1,2}=-\left(10-r_{1,2}\right) x_{1,2}-11 y_{1,2}+\left(10+r_{1,2}\right) N_{1,2} \\
-\left(x_{1,2}+N_{1,2}\right) z_{1,2}+\frac{\varepsilon}{2}\left(y_{2,1}-y_{1,2}\right), \\
\dot{z}_{1,2}=\frac{1}{2} y_{1,2}-\frac{8}{3} z_{1,2},
\end{gathered}
$$

where $N=\sqrt{x^{2}+y^{2}}$. As before, $\varepsilon$ measures the coupling strength. The system parameter $r$ has been given values $r_{1}$ $=36$ and $r_{2}=60$, the two uncoupled systems being chaotic and different at these parameters.

A study similar to those shown above, including the calculation of $\Omega_{1,2}(\varepsilon)$ and $r_{2,1}(\varepsilon)$ from $z_{1,2}(t)$, as well as the Lyapunov spectrum $\lambda_{i}(\varepsilon)$, is displayed in Fig. 10. The varia- 
tion of $\Omega_{1,2}$ shows a neat phenomenon of PS as $\Omega_{1}$ and $\Omega_{2}$ tend to a common value of $\Omega_{1,2} \approx 12.5$. This transition occurs at $\varepsilon \approx 2.1$. However, the Lyapunov spectrum displays a fourth exponent becoming negative well before PS is reached. This is understood if one looks at the behavior of $r_{2,1}$, which shows a development of AES, which for $\varepsilon$ $\lesssim 1.5$ is mild but increases very fast for $\varepsilon \geq 1.5$. At the same time the frequency difference $\Omega_{2}-\Omega_{1}$ gradually tends to zero giving rise to PS. The main differences with the circuit of Chua is that in this case, only Regime II is observable, chaos is not destroyed shortly after PS has been reached, and the synchronization states with PS and AES combined are persistent well above the transition to PS. Moreover, the correlation between the two signals, $r$, becomes important for $\varepsilon \gtrsim 2.2$. All this indicates an important degree of correlation between the two time series $z_{1}(t)$ and $z_{2}(t)$, once the two forms of synchronization have been developed.

An example of the highly correlated states resulting from AES and PS combined is shown in Fig. 11 by means of the plot of $P_{1,2}(\omega)$ and $z_{1,2}(t)$ at $\varepsilon=2.4$, which is above the onset of PS. The power spectra [Figs. 11(a) and 11(b)] show how new broad peaks have appeared at very small frequencies of $\omega_{1,2}^{(0)} \approx 1.5$, while the dominant peaks have become broader and are locked at $\omega_{1,2}^{(M)} \approx 12.5$. The plots for the evolution of the system variables, $z_{1,2}(t)$, given in Figs. 11(c) and 11(d) show the synchronization of the phase combined with the synchronization of the envelopes of the amplitude for two signals that otherwise are different and chaotic.

\section{DISCUSSION AND CONCLUSIONS}

The manifestation of AES is somehow subtle; therefore, it might pass unnoticed unless attention is paid to the Lyapunov exponents, and to the peak-to-peak dynamics [19] of the systems. Moreover, as it develops steadily from $\varepsilon$ $\geq 0$, it might be extremely weak in the particular parameter region studied. Furthermore, it happens that the development of AES is compatible with PS in the sense that once the new frequencies $\omega_{1,2}^{(0)}$ have developed, the dominant frequencies in the power spectra of each system may rearrange so that $\Omega_{1}$ and $\Omega_{2}$ move to the get locked in a way similar to that described above for two mutually coupled Rossler oscillators (and by Parlitz et al. [4] for a driven Rossler system). This shows that, if the focus is on PS, and AES is disregarded, in some cases one may be faced with a puzzle in which a Lyapunov exponent becomes negative before PS occurs. In fact, it is possible to find in the literature on PS statements of concern about the applicability of the Lyapunov exponents to study PS $[5,12]$. AES may be the piece needed to solve these puzzles, and therefore a relevant element needed for a thorough understanding of the synchronization phenomena.

The Van der Pol-Duffing oscillator and the Rossler system studied here and in Ref. [2], respectively, appear as two extreme cases in which only one of the two forms of synchronization is easily noticeable. When observed in Fourier space, the dynamic mechanism for AES happens to be the mutual excitation of new frequencies (with its harmonics), while the mechanism for PS is the rearrangement of the power spectra structures around the dominant frequencies. As these mechanisms are simple and appear quite general, one has to expect a certain degree of variety on the dynamics to be obtained from two coupled chaotic oscillators. In particular, both AES and PS may appear combined in many systems as shown here by means of the example of two coupled Chua circuits, and of two coupled Proto-Lorenz systems. Even in this case the phenomenology observed has found to bear some diversity, including the early development of AES without PS, the development of PS and AES simultaneously, as well as differences in the persistence of the combined highly synchronized state achieved in this last case.

In summary, the dynamics of two different Van der PolDuffing oscillators mutually coupled shows that, as a result of the coupling, a new common frequency that is located well below the frequencies of the phase rotations around the centers of the attractors is induced in the two coupled oscillators. The increase of the coupling results in a feedback process between the two systems that develops and enhances this new common frequency, giving rise to the form of synchronization named AES in this paper to emphasize that it concerns to the envelope of the amplitude of the main oscillation rather than to its frequency. Because this phenomenology is so neatly displayed by the Van der Pol-Duffing oscillator, the study presented in Secs. II and III happens to be a paradigmatic example of a synchronization behavior that, because of its own nature should be present in other systems, although possibly mixed with or hidden by other forms of synchronization. This has been shown indeed to be the case by means of the study of the model of Rossler, the circuit of Chua, and the Proto-Lorenz system. At a speculative level, one might think that AES may be useful in scientific and technical applications, and anyway, it is an effect to be considered when chaotic oscillators are coupled to each other.

\section{ACKNOWLEDGMENT}

This research has been supported by DGI through Project No. BFM2000-0606.
[1] H. Fujisaka and T. Yamada, Prog. Theor. Phys. 56, 32 (1983).

[2] M.G. Rosenblum, A.S. Pikovsky, and J. Kurths, Phys. Rev. Lett. 76, 1804 (1996).

[3] L.M. Pecora, and T.L. Carroll, Phys. Rev. Lett. 64, 821 (1990).

[4] U. Parlitz, L. Junge, W. Lauterborn, and L. Kocarev, Phys. Rev. E 54, 2115 (1996).
[5] A.S. Pikovsky, M.G. Rosenblum, G.V. Osipov, and J. Kurths, Physica D 104, 219 (1997).

[6] C. Schafer, M.G. Rosenblum, H.H. Abel, and J. Kurths, Phys. Rev. E 60, 857 (1999).

[7] F. Mormann, K. Lehnertz, P. David, and C.E. Elger, Physica D 144, 358 (2000). 
[8] G.P. King and S.T. Gaito, Phys. Rev. A 46, 3092 (1992).

[9] O.E. Rossler, Phys. Lett. 57A, 397 (1976).

[10] T. Matsumoto, L.O. Chua, and M. Komuro, IEEE Trans. Circuits Syst. 32, 797 (1985).

[11] R. Miranda and E. Stone, Phys. Lett. A 178, 105 (1993).

[12] J.-W. Shuai and D.M. Durand, Phys. Lett. A 264, 289 (1999).

[13] M.G.M. Gomes and G.P. King, Phys. Rev. A 46, 3100 (1992).

[14] W.H. Press, B.P. Flannery, S.A. Teukolsky, and W.T. Vetterling, Numerical Recipes (Cambridge University Press, Cambridge, U.K., 1990).
[15] N.F. Rulkov, M.M. Sushchik, L.S. Tsimring, and H.D.I. Abarbanel, Phys. Rev. E 51, 980 (1995).

[16] M.G. Rosenblum, A.S. Pikovsky, and J. Kurths, Phys. Rev. Lett. 78, 4193 (1997).

[17] L.O. Chua, L. Kocarev, K. Eckert, and M. Itoh, Int. J. Bifurcation Chaos Appl. Sci. Eng. 2, 705 (1992).

[18] E.N. Lorenz, J. Atmos. Sci. 20, 130 (1963).

[19] M. Candaten and S. Rinaldi, Int. J. Bifurcation Chaos Appl. Sci. Eng. 10, 1805 (2000). 